\title{
Using mobile-device sensors to teach students error analysis
}

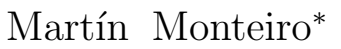 \\ Universidad ORT Uruguay \\ Cecila Stari, Cecila Cabeza, and Arturo C. Marti \\ Instituto de Física, Facultad de Ciencias, \\ Universidad de la República, Iguá 4225, Montevideo, 11200, Uruguay
}

(Dated: September 16, 2020)

\begin{abstract}
Science students must deal with the errors inherent to all physical measurements and be conscious of the need to expressvthem as a best estimate and a range of uncertainty. Errors are routinely classified as statistical or systematic. Although statistical errors are usually dealt with in the first years of science studies, the typical approaches are based on manually performing repetitive observations. Our work proposes a set of laboratory experiments to teach error and uncertainties based on data recorded with the sensors available in many mobile devices. The main aspects addressed are the physical meaning of the mean value and standard deviation, and the interpretation of histograms and distributions. The normality of the fluctuations is analyzed qualitatively comparing histograms with normal curves and quantitatively comparing the number of observations in intervals to the number expected according to a normal distribution and also performing a Chi-squared test. We show that the distribution usually follows a normal distribution, however, when the sensor is placed on top of a loudspeaker playing a pure tone significant differences with a normal distribution are observed. As applications to every day situations we discuss the intensity of the fluctuations in different situations, such as placing the device on a table or holding it with the hands in different ways. Other activities are focused on the smoothness of a road quantified in terms of the fluctuations registered by the accelerometer. The present proposal contributes to gaining a deep insight into modern technologies and statistical errors and, finally, motivating and encouraging engineering and science students.
\end{abstract}




\section{INTRODUCTION}

In many experimental situations when a measurement is repeated -for example when we measure a time interval with a stopwatch, the landing distance of a projectile or a voltage with a digital multimeter-- successive readings give slightly different results under identical conditions. This occurs beyond the care we take to always launch the ball exactly the same way or to connect the components of the circuit so that they are firmly attached. In effect, this phenomenon occurs in the real world because most measurements present statistical uncertainties ${ }^{12}$. When facing repeated observations with different results it is natural to ask ourselves which value is the most representative and what confidence level can we have in that value. The International Standard Organization (ISO) ${ }^{3}$ (see also ${ }^{45}$ ) defines the errors evaluated by means of the statistical analysis of a series of observations as type A, in contrast with other sources of errors that are systematic and defined as type B. The evaluation of the latter is estimated using all available non-statistical information such as instrument characteristics or the individual judgment of the observer. In this work, we focus on the teaching of statistical errors in the first years of science and engineering studies using modern sensors.

The study of error analysis and uncertainties plays a prominent role in the first years of all science courses. On this matter, AAPT recommends ${ }^{6}$ that students should be able to use statistical methods to analyze data and should be able to critically interpret the validity and limitations of the data displayed. In general, a physicist must be able to design a measurement procedure, select the equipment or instruments, perform the process and finally express the results as the best estimate and its of uncertainty. Perhaps the most important message is to persuade students that any measurement is useless unless a confidence interval is specified. It is expected that, after finishing their studies, students are able to discuss whether a result agrees with a given theory and, if it is reproducible, or to distinguish a new phenomenon from a previously known one. With this objective, various experiments are usually proposed in introductory laboratory courses ${ }^{7-12}$. These experiments usually involve a great amount of repetitive measurements such as dropping small balls ${ }^{12}$, measuring the length of hundreds or thousands of nails using a vernier caliper ${ }^{9}$ or randomly sampling an alternating current source ${ }^{10}$. The measurements obtained are usually examined from a statistical viewpoint plotting, histograms, calculating mean values and standard deviations 
and, eventually, comparing them with those expected from a known distribution, typically a normal distribution. Although these experiments are illustrative, most of them are tedious and do not adequately reflect the present state of the art.

In contrat with the expectable learning outcomes mentioned above, recent studies $13-15$ report several difficulties associated with error analysis among students. The study by Séré et $a l^{13}$ highlighted the lack of understanding of the need to make several measurements, the poor insight into the notion of confidence intervals or the inability to distinguish between random and systematic errors. Another investigation ${ }^{14}$ remarked the inconsistency of the common view of students with generally accepted scientific models. On many occasions, the student's model of thinking is close to a point paradigm as opposed to a more elaborated probabilistic interpretation of the measurements. A reseach-based assesment showed that although the impact of introductory laboratory courses was positive, only a relatively small percentage of students developed a deeper understanding of measurement uncertainty 16 .

The fluctuations present in the sensors of modern mobile devices give rise to an alternative approach to teaching error analysis. Indeed, most smartphones and tablets come equipped with several built-in sensors such as accelerometers, magnetometers, proximeters or ambientlight sensors. Several Physics experiments using these sensors have been proposed in recent years (see for example $\sqrt{17-[9}$ ). In almost all the experiments, only mean values are taken into consideration; however, due to their sensitivity, sensor readings also display statistical fluctuations. Although being detrimental in many situations, these fluctuations can be used favorably to illustrate basic concepts related to the statistical treatment of measurements. Using these sensors, it is certainly possible to acquire hundreds or thousands of repeated values of a physical magnitude in a few seconds and analyze them in the mobile device or in a PC. We propose here a set of laboratory activities to teach error analysis and uncertainties in introductory Physics laboratories based on the fluctuations registered by mobile-device sensors. In the next Section we describe the basic set of activities while Section III focused on other applications that take into account sensor fluctuations in non-standard situations. Finally, in Section IV we present the summary and conclusion. 


\section{A LABORATORY BASED ON MOBILE DEVICES}

Among all the sensors, accelerometers, capable of measuring the acceleration of the device in the three independent spatial directions, are the most ubiquitous in mobile devices. Though it is possible to use accelerometers or anyone of the others or even more than one sensor simultaneously here, for the sake of clarity, the proposed experiments are mainly based on the $z$ component of the acceleration $a_{z}$, defined as perpendicular to the screen. As a general rule, the characteristics of the sensors can be found using specific applications (apps) or looking for datasheets in the internet. The range (difference between the maximum and minimum value that it is capable of measuring) and the resolution (minimum difference that the sensor can register, which is sometimes incorrectly termed as accuracy) of several sensors are summarized in Table I. It is worth remarking that, although being universally known as accelerometers, in fact, they are force sensors 20121 . Indeed, a device standing on a table would register a value close to the gravitational acceleration in the vertical axis while a free-falling device would register a value close to zero in the same axis.

\begin{tabular}{|c|c|c|c|}
\hline Phone & Sensor & Range $\left(\mathrm{m} / \mathrm{s}^{2}\right)$ & Resolution $\left(\mathrm{m} / \mathrm{s}^{2}\right)$ \\
\hline Samsung Galaxy S7 & K6DS3TR & \pm 78.4532 & 0.0023942017 \\
LG G3 & LGE & \pm 39.226593 & 0.0011901855 \\
Nexus 5 & MPU-6515 & \pm 19.613297 & 0.0005950928 \\
iPhone 6 & MPU-6700 & - & - \\
Samsung J6+ & LSM6DSL & \pm 39.2266 & 0.0011971008 \\
Xiaomi Redmi Note7 & ICM20607 & \pm 78.4532 & 0.0011901855 \\
Samsung Galaxy S9 & LSM6DSL & \pm 78.4532 & 0.0023942017 \\
Samsung A20S & ICM40607 & \pm 78.4532 & 0.0023956299 \\
\hline
\end{tabular}

TABLE I. Range and resolution of several common mobile devices obtained with the Androsensor app. Notice that sensors can usually operate within different ranges $\pm 2 g, \pm 4 g, \pm 8 g, \ldots$, which are set by the mobile-device manufacturer. The resolution depends on the choice of range.

In the case of the iPhone the manufacturer does not provides this information.

In general, a specific piece of software, or an app, is necessary. Digital stores offer many apps that are able to communicate with the sensors. In particular, Physics Toolbox Suite ${ }^{22}$, 
Androsensor and PhyPhox ${ }^{23}$, whose screenshots are shown in Fig. 1, are suitable for the experiments proposed here. Using these apps it is possible to select the relevant sensors, and to setup the parameters such as the duration of the time series and the sampling frequency. The registered data can be analyzed directly on the smartphone screen or transferred to the cloud and studied on a PC using a standard graphics package. Others useful characteristics of these apps are the delayed execution and the remote access via $w i$ - $f$ or browser. These capabilities allow the experimenter to avoid touching or pushing the mobile device once the experiments has started.
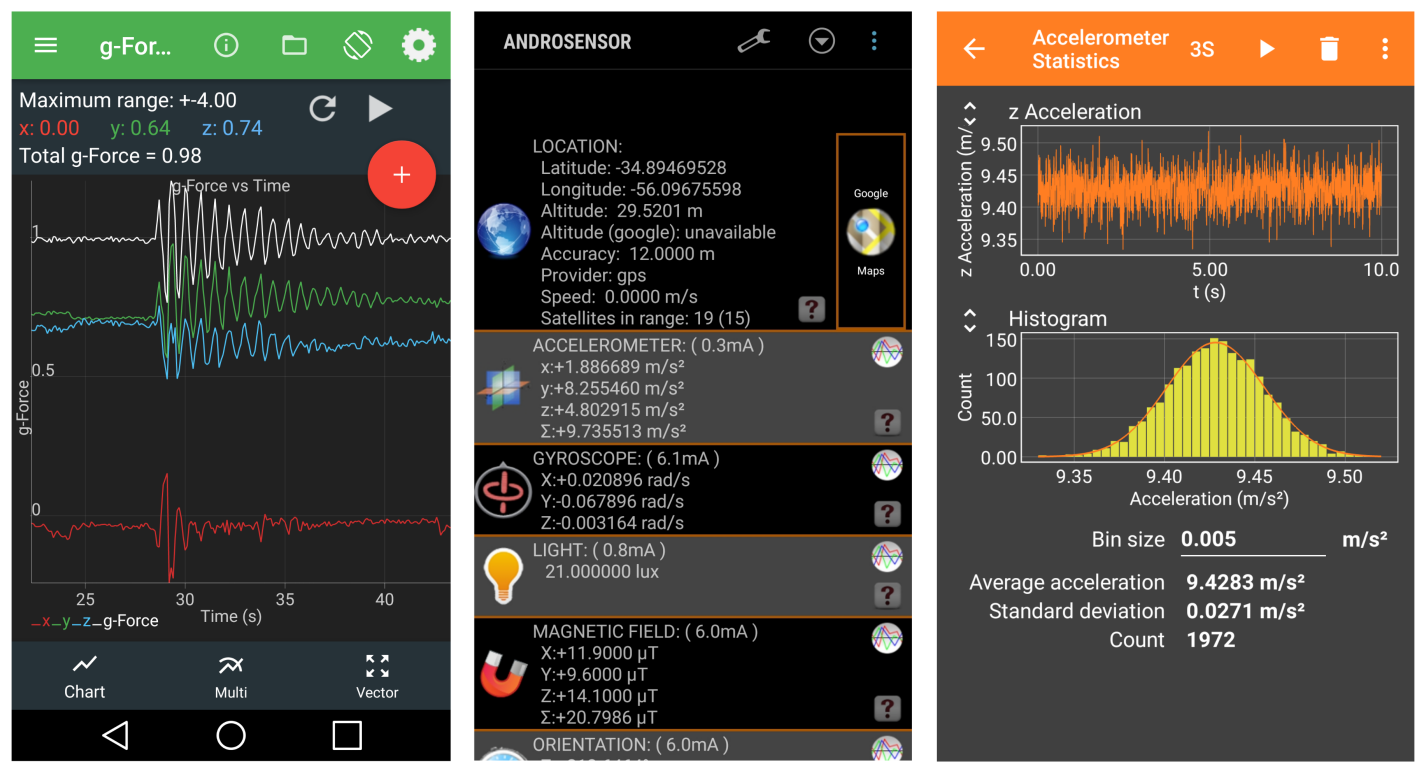

FIG. 1. Screenshots of three suitable apps: Physics Toolbox suite (left), Androsensor (center), Phyphox (right). The right panel shows a Phyphox screenshot of the experiment Statistical Basics including a temporal series of the vertical component of the acceleration (top) and the corresponding histogram (bottom) overlapped with a Gaussian curve with the same mean and standard deviation indicated in the image.

\section{A. Normal distribution of the sensors' fluctuations}

The first experiment consists of recording the fluctuations of the vertical component of the accelerometer sensor with the mobile device in three different situations: laid on a table, hand-held and resting on another smartphone playing a $600 \mathrm{~Hz}$ pure tone. In all the cases, we 
choose, unless stated otherwise, a delay of $3 \mathrm{~s}$ and register $a_{z}$ for $30 \mathrm{~s}$. The delay is important in order to avoid touching the device when the register starts and thus introducing spurious values. Let us denote $N$ the number of measurements registered by the sensor, $\bar{a}_{z}$ the mean value and $\sigma_{a_{z}}$ the standard deviation.

The results of the experiment are summarized in Fig. 2 in which the top panels display the temporal series, $a_{z}(t)$, and the bottom panels show the histograms using the same respective colors. In all the cases the accelerations fluctuate stationarily around mean values. Although these values are close to the well-known value of the gravitational acceleration, they are slightly different and they are not expected to represent a measure of that magnitude. This is due to several reasons, for instance it depends on the horizontalitly of the table or the hand or also on the calibration of the sensor. It is interesting for students to check that the mean value changes when the device is laid on a table with the screen pointing upwards or downwards. Another possible, and equivalent, alternative (not shown here) consists of plotting $a_{x}(t)$ or $a_{y}(t)$ which exhibit similar temporal evolutions and histograms but fluctuating around a value close to $0 \mathrm{~m} / \mathrm{s}^{2}$.

The differences in the intensity of the fluctuations exhibited in the three mentioned situations are evident in the top panels of Fig. 2. The intensity is clearly larger when the smartphone is hand-held (red) or under the influence of the $600 \mathrm{~Hz}$ tone (green) in comparison with the smartphone on a table (blue). The standard deviation of each series, indicated in the legend boxes, is clearly related to the intensity of the fluctuations. This observation substantiates the use of the standard deviation in the framework of the applications proposed in Section III.

A relevant aspect to study is the distribution of the fluctuations and how it compares with the normal distribution. The firt approach to testing the normality of the distribution is qualitative. In the bottom panels (Fig. 2), the histograms are compared with normal (Gaussian) functions with the same mean values and standard deviation and the vertical scale adjusted so that the area under the normal curve and the sum of the bins of the histogram are equal. It can be observed that the histograms and normal functions agree very well in the cases of the blue and red curves. By increasing the number of samples $N$ and simultaneously decreasing the width of the bins, it is possible to see that the agreement improves even more (not shown here). Contrarily, in the green case, the pure tone breaks the normality of the distributions as it is clearly revealed by the disagreement between the 

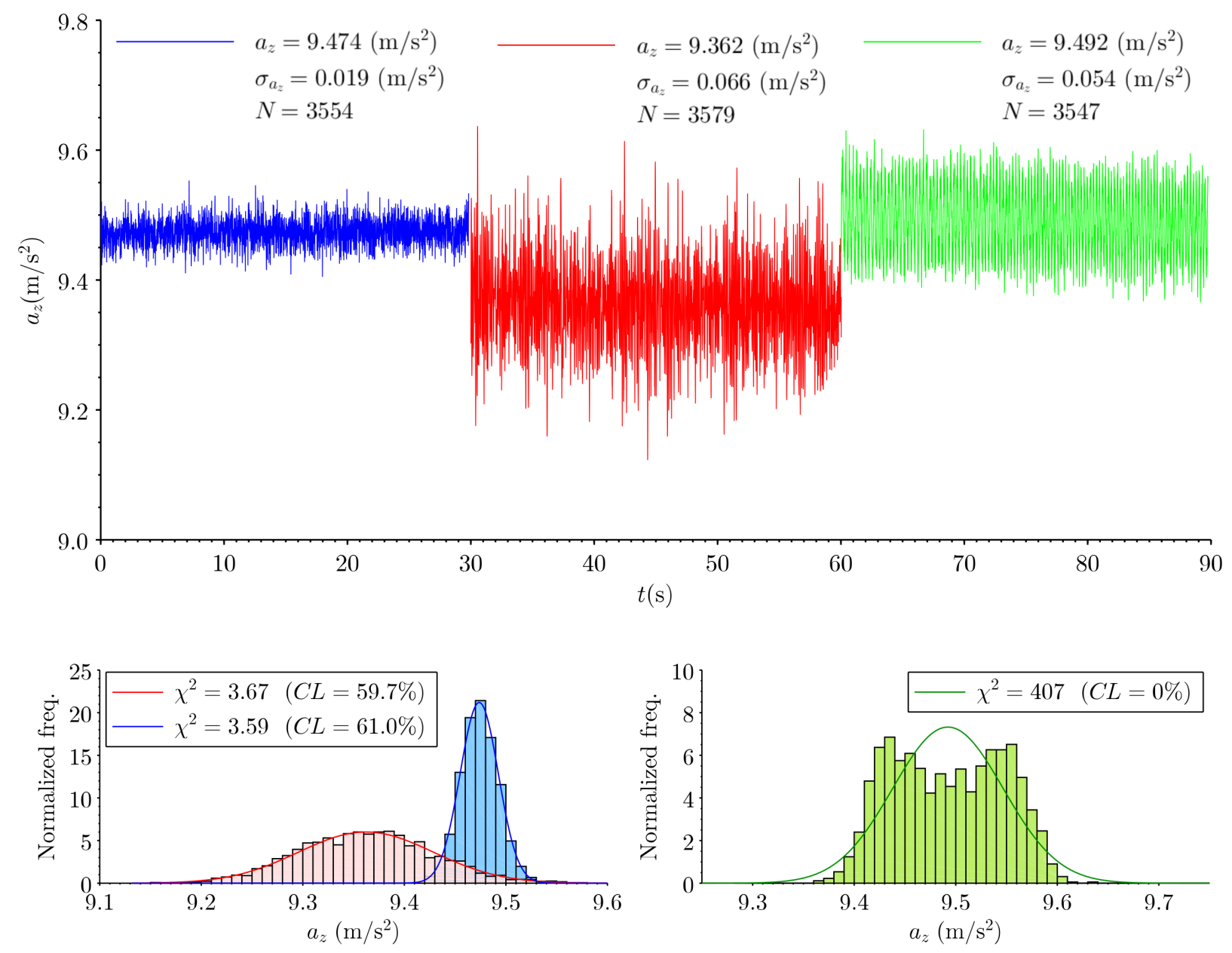

FIG. 2. Fluctuations registered by the accelerometer. The top panels display $a_{z}(t)$ with the device horizontal in three different situations: laid on a table (blue), hand-held (red) and resting on another smartphone playing a $600 \mathrm{~Hz}$ pure tone (green). The smartphone was the LG-G3 with a $\Delta t=0.004 \mathrm{~s}$ sampling period. The bottom panels display the histograms and the continuous lines with the same color are normal (Gaussian) functions with same mean value, standard deviation and normalization. Legend boxes indicate $\chi^{2}$ values, calculated with 8 bins, and the corresponding confidence levels (CL).

histogram and normal curve.

The second and more quantitative approach to verifying the normality of the distributions is given by the comparison of the fraction of observations in a given interval around the mean value and the expected percentage according to a normal distribution. Table II displays these percentages for the experiment depicted in Fig. 2, It can be seen that, in agreement with the 
qualitative test, the observed and expected percentages are quite similar when the device is on the table or hand-held where they present considerable divergences under the influence of the $600 \mathrm{~Hz}$ tone.

\begin{tabular}{|c|c|c|c|c|}
\hline Experiment & Table & Hand & Speaker & Theoretical \\
\hline $\mathrm{N}$ & 3554 & 3579 & 3547 & - \\
\hline $\bar{x} \pm \sigma\left(\mathrm{m} / \mathrm{s}^{2}\right)$ & $9.474 \pm 0.019$ & $9.362 \pm 0.066$ & $9.492 \pm 0.054$ & - \\
\hline$(\bar{x}-\sigma, \bar{x}+\sigma)$ & $69.3 \%$ & $67.9 \%$ & $58.2 \%$ & $68.2 \%$ \\
$(\bar{x}-2 \sigma, \bar{x}+2 \sigma)$ & $95.2 \%$ & $95.5 \%$ & $99.2 \%$ & $95.4 \%$ \\
$(\bar{x}-3 \sigma, \bar{x}+3 \sigma)$ & $99.7 \%$ & $99.7 \%$ & $100.0 \%$ & $99.7 \%$ \\
\hline
\end{tabular}

TABLE II. Fraction of observations in intervals around the mean defined in units of the standard deviation compared with the expected number according to a normal distribution. Each column corresponds to each of the temporal series plotted in Fig. 2.

To gain further insight into the normality of the distributions, a chi-squared test comparing the difference between the number of observations measured and expected in each bin ${ }^{1}$, was performed. Each $\chi^{2}$ valued can be associated with a confidence level that determines the rejection of the hypothesis of normal distribution. Clearly in the blue and red cases the $\chi^{2}$ test indicates the compatibility of the normal distribution hypothesis while in the green case this hypothesis must be rejected.

\section{B. Resolution in digital sensors}

By zooming in on the temporal series displayed in Fig. 2, it can be seen that the sensor values do not take continuous values, but only a discrete set is possible. This is more evident in the experiment displayed in Fig. 3 where the horizontal axis has been zoomed out in the left panel, and a horizontal histogram with the same values is shown in the right panel. The difference between the discrete values in the vertical axis is the resolution of the instrument, that is, the minimum difference that the sensor can register. This is typical of digital instruments, where a continuous magnitude (such as acceleration, in this case) is transformed by a sensor into an analog electrical signal, which is in turn transformed by an analog-to-digital converter (ADC) into a digital signal which can only take certain 


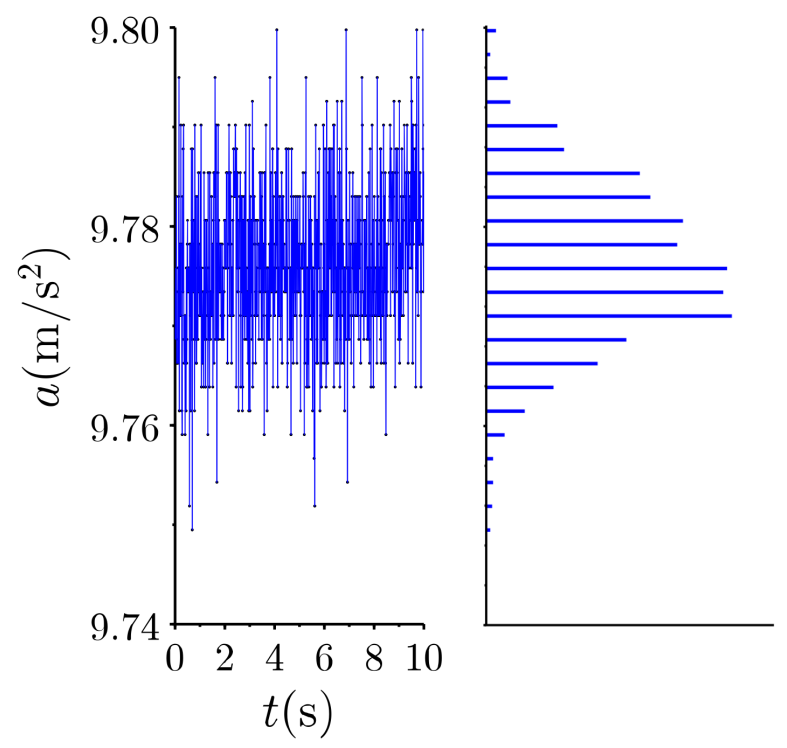

FIG. 3. Discrete nature of the sensor data. The horizontal axis in the left panel was zoomed out to emphasize the discrete nature of the accelerometer values. The right panel shows the same values in a horizontal histogram with the same vertical scale.

discrete values. In this case, the acceleration sensor of the Samsung S7 is a K6DS3TR and its resolution, indicated in Table $\mathrm{I}$, is $\delta=0.0023942017 \mathrm{~m} / \mathrm{s}^{2}$ which corresponds exactly to the difference between consecutive acceleration values.

The resolution of the sensor, $\delta$, is the quotient between the range, $2 R$, and the number of different values that the sensor can register, $2^{n}$,

$$
\delta=\frac{2 R}{2^{n}}
$$

where $n$ is the number of bits of the sensor and the factor 2 stands because it registers not only positive measures, but also negative accelerations. Taking into consideration Table I] it can be determined that this sensor is capable of measuring $2 R / \delta=65536$ different values and since $65536=2^{16}$, this means that it is a 16 -bit sensor, which can be easily verified on the data sheets.

\section{Standard error and optimal number of measurements}

The standard deviation, if $N$ is large enough, is characteristic of the set of all the possible observations whereas the standard error, or standard deviation of the mean, generally defined 


\begin{tabular}{|c|c|c|c|c|c|c|c|c|c|c|}
\hline$N$ & 563 & 1156 & 1746 & 2348 & 2941 & 3535 & 4166 & 4733 & 5327 & 5919 \\
\hline$\sigma_{a_{z}}\left(\mathrm{~m} / \mathrm{s}^{2}\right)$ & 0.020 & 0.019 & 0.018 & 0.019 & 0.020 & 0.019 & 0.019 & 0.019 & 0.019 & 0.020 \\
\hline
\end{tabular}

TABLE III. Standard deviations of $a_{z}(t)$ corresponding to several experiments under identical conditions but with different number of measurements.

as $\sigma\left(\overline{a_{z}}\right)=\sigma_{a_{z}} / \sqrt{N}$ represents the margin of uncertainty of the mean value obtained in a particular set of measurements ${ }^{1}$. The result of a specific measurement is usually expressed as $\bar{a}_{z} \pm \sigma\left(\overline{a_{z}}\right)$ representing the best estimate and the confidence in that value. In Table. III the standard deviation is shown as a function of $N$. As mentioned above, it is clear from that data that $\sigma_{a_{z}}$ is nearly constant and, as a consequence, $\sigma\left(\overline{a_{z}}\right)$ is proportional to $N^{-1 / 2}$.

The choice of $N$ in a specific experiment is a delicate question. Indeed, if we could repeat the measurements infinite times the standard error would vanish and we could achieve a perfect knowledge of the best estimate. However, as the decrease of the standard error with the number of observations is slow, it is impractical to increment this number excesively. A common criterion is to take a number of measurements, often referred as the optimal number of measurements, $N_{\text {opt }}$, such that the statistical uncertainty is of the same order as the systematic (or type B) errors. Here, in the absence of other sources of systematic errors, the standard error should be of the same order as the resolution of the digital instrument: $\sigma\left(\overline{a_{z}}\right)=\sigma_{a_{z}} / \sqrt{N_{\text {opt }}} \sim \delta$. In the experiment depicted in Table III with a LG G3, the resolution is $\delta=0.0012 \mathrm{~m} / \mathrm{s}^{2}$, therefore $N_{\text {opt }} \sim 250$.

\section{OTHER APPLICATIONS}

In this Section we propose a couple of activities in which the knowledge of the fluctuations measured by a sensor can contribute to quantify another magnitude.

\section{A. The steady hand game}

An interesting experiment is to study the intensities of the fluctuations depending on the way in which the experimenter holds his/her device. This activity can be adapted for a group of students as a challenge consisting of trying to hold the device as steadily as 


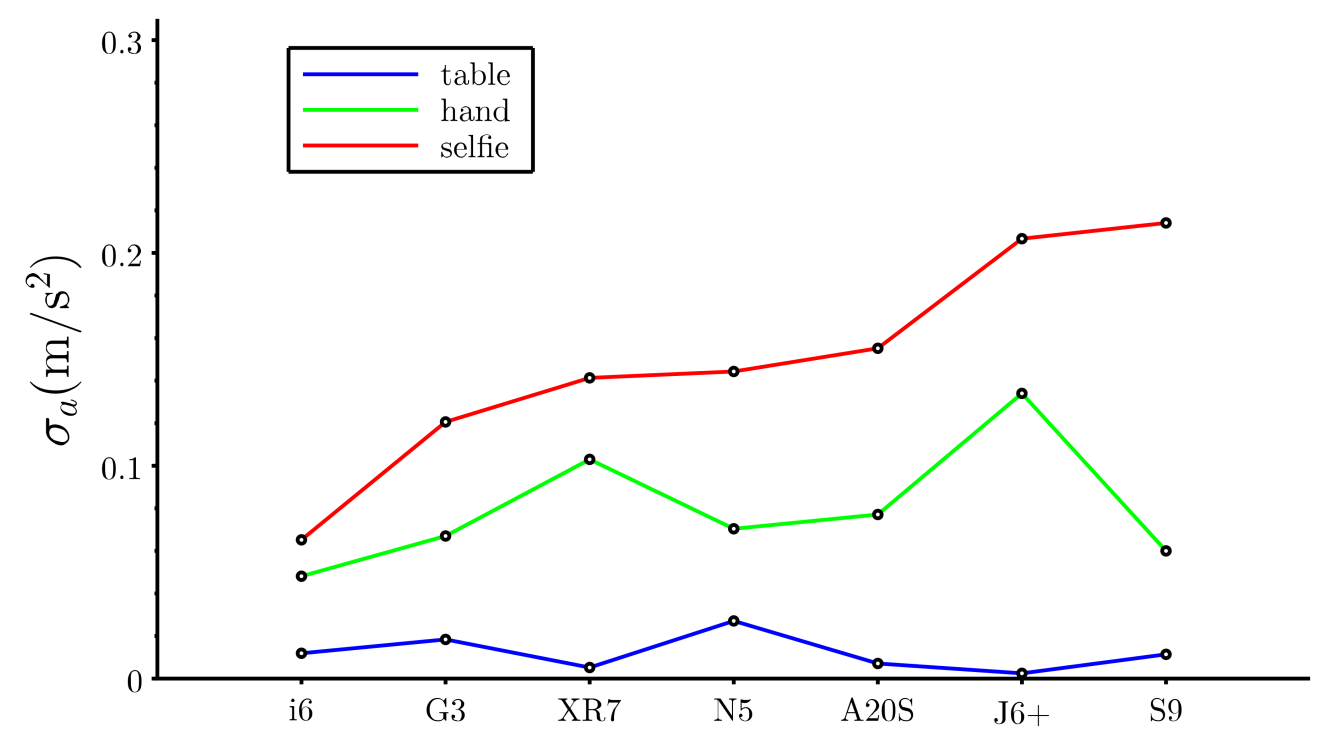

FIG. 4. Comparative table of the standard deviation $\sigma$ for different mobile devices in different activities as a function of the different models (see Table II). Lines are guides for the eyes

possible. Another possibility (not recommended by the authors) is to study the fluctuations of the gait of a pedestrian as a function of the alcohol beverage intake similar $t \mathrm{~d}^{24}$.

The steadiness of the device is quantified by the standard deviation of a given temporal series. The intensities of the fluctuations in different situations and for different sensors are displayed in Fig. 4. It is evident from these values that the mobile device on the table exhibits in all the cases less fluctuations than when the device is held by the experimenter. Moreover a more stable position is achieved by keeping the device close to the trunk as opposed to the classical selfie position. Another point worth mentioning is that the intensity of the fluctuations depends on the specific sensor but exhibits in all cases the same trends mentioned above. Several interesting extensions to this experiment can be proposed: the dependence on the characteristics of the experimenter (age, training, concentration). The origin of the fluctuations, mechanical or electronical, can be considered in case of having an antivibration table. When using interferometric methods a precise calibration of the mobile device sensor could also be performed. 


\section{B. The smartphone as a way to assess road quality}

Recently, smartphones' sensors were proposed to assess road quality 25 . In this activity, which can be performed outdoors, students can assess the quality of a road. A means of transport, in this case a car, is employed under similar conditions (speed), but other possibilities, such as a bike, are equally feasible. The intensities of the fluctuations traveling by car on different roads are listed in Table IV. To get an insight of the fluctuations attributable to the road the noise with the car stopped and the engine idle is indicated in the first road. A similar measurement performed in a flying aircraft is included solely for the sake of comparison. This activity can be extended to evaluate comfort in any other means of transport media, for example, elevators.

\begin{tabular}{|l|l|l|l|l|}
\hline Situation & $\mathrm{N}$ & $\sigma_{\mathrm{G} 3}\left(\mathrm{~m} / \mathrm{s}^{2}\right)$ & $\mathrm{N}$ & $\sigma_{\mathrm{XR} 7}\left(\mathrm{~m} / \mathrm{s}^{2}\right)$ \\
\hline Engine idle & 1181 & 0.3818 & 4984 & 0.0352 \\
Smooth pavement & 1200 & 1.3487 & 4974 & 0.5642 \\
Stone pavement & - & - & 4952 & 1.1491 \\
Aircraft & 1999 & 0.4374 & - & - \\
\hline
\end{tabular}

TABLE IV. Assessment of the quality of different roads. Standard deviation of $a_{z}$ while the device is on the floor of the car with the screen orientated upwards.

\section{SUMMARY AND CONCLUSION}

The activities discussed above were successfully proposed at our university to freshman Engineering and Physics students. In previous laboratory experiments students already knew the usefulness of the sensor to study several phenomena in which the noise was a factor to avoid. However, when sensors were proposed to study fluctuations, they were surprised to discover a kind of underlying world. Despite having gone through statistical topics in several courses, the normal distribution appearing as an experimental fact rather than a mathematical consequence, was original. It is worth discussing, how the distributions of the other sensors change. For example, magnetometer fluctuations, significantly influenced by motors or ferromagnetic material in the vicinity, do not follow normal distributions. Several 
challenges can be proposed in relation to sports, comfort evaluations or quality control.

The main conclusion is that modern mobile-device sensors are useful tools for teaching error analysis and uncertainties. In this work we proposed several activities that can be performed to teach uncertainties and error analysis using digital instruments and the builtin sensors included in modern mobile devices. It is straightforward to obtain experimental distributions of fluctuations and compare them with the expected ones. It is shown that the distributions usually obey normal (Gaussian) statistics; however, it is easy to obtain non normal distributions. The role of noise intensity, spreading or narrowing the distributions open up the possibilities of new applications. Holding the mobile device in different ways also gives an idea of how firmly it is held. Registering acceleration values in a car can assess the smoothness of a road. In this approach, the lengthy and laborious manipulations of traditional approaches based on repetitive measurements, are avoided allowing teaching to focus on the fundamental concepts. These experiments could contribute to motivating students and showing them the necessity of considering uncertainty analysis. Several possible extensions related to non-normal statistics can be considered, such as Poison distribution ${ }^{8}$, distribution of maxima, Chauvenet criterion 26 , or Benford law 27 .

\section{ACKNOWLEDGMENT}

The authors would like to thank PEDECIBA (MEC, UdelaR, Uruguay) and express their gratitude for the grant Fisica Nolineal (ID 722) Programa Grupos I+D CSIC 2018 (UdelaR, Uruguay).

* monteiro@ort.edu.uy

$\dagger$ marti@fisica.edu.uy

1 John Taylor. Introduction to error analysis, the study of uncertainties in physical measurements. University Science Books, 1997.

2 Ifan Hughes and Thomas Hase. Measurements and their uncertainties: a practical guide to modern error analysis. Oxford University Press, 2010.

3 OIML ISO. Guide to the expression of uncertainty in measurement (gum). Geneva, Switzerland, 1995. 
4 Barry N Taylor, Peter J Mohr, and M Douma. The nist reference on constants, units, and uncertainty. available online from: physics. nist. gov/cuu/index, 2007.

5 Bureau International des Poids et Mesures. Evaluation of measurement data-guide to the expression of uncertainty in measurement, 2008.

6 Joseph Kozminski, Heather Lewandowski, Nancy Beverly, Steve Lindaas, Duane Deardorff, Ann Reagan, Richard Dietz, Randy Tagg, Jeremiah Williams, Robert Hobbs, et al. Aapt recommendations for the undergraduate physics laboratory curriculum. American Association of Physics Teachers, 29, 2014.

7 I. M. Meth and L. Rosenthal. An experimental approach to the teaching of the theory of measurement errors. IEEE Transactions on Education, 9(3):142-148, 1966.

8 E. Mathieson and T. J. Harris. A student experiment on counting statistics. American Journal of Physics, 38(10):1261-1262, 1970.

9 P. C. B. Fernando. Experiment in elementary statistics. American Journal of Physics, 44(5):460463, 1976.

10 Arvind, P. S. Chandi, R. C. Singh, D. Indumathi, and R. Shankar. Random sampling of an alternating current source: A tool for teaching probabilistic observations. American Journal of Physics, 72(1):76-82, 2004.

11 Tadeusz Wibig and Punsiri Dam-o. 'hands-on statistics' - empirical introduction to measurement uncertainty. Physics Education, 48(2):159-168, feb 2013.

12 K K Gan. A simple demonstration of the central limit theorem by dropping balls onto a grid of pins. European Journal of Physics, 34(3):689-693, mar 2013.

13 Marie-Geneviève Séré, Roger Journeaux, and Claudine Larcher. Learning the statistical analysis of measurement errors. International Journal of Science Education, 15(4):427-438, 1993.

14 Saalih Allie, Andy Buffler, Bob Campbell, Fred Lubben, Dimitris Evangelinos, Dimitris Psillos, and Odysseas Valassiades. Teaching measurement in the introductory physics laboratory. The Physics Teacher, 41(7):394-401, 2003.

15 MF Chimeno, MA Gonzalez, and J RAMOS Castro. Teaching measurement uncertainty to undergraduate electronic instrumentation students. International Journal of Engineering Education, 21(3):525-533, 2005.

16 Trevor S. Volkwyn, Saalih Allie, Andy Buffler, and Fred Lubben. Impact of a conventional introductory laboratory course on the understanding of measurement. Phys. Rev. ST Phys. 
Educ. Res., 4:010108, May 2008.

17 Rebecca Vieyra, Chrystian Vieyra, Philippe Jeanjacquot, Arturo Marti, and Martín Monteiro. Five challenges that use mobile devices to collect and analyze data in physics. The Science Teacher, 82(9):32-40, 2015.

18 Katrin Hochberg, Jochen Kuhn, and Andreas Müller. Using smartphones as experimental tools - effects on interest, curiosity, and learning in physics education. Journal of Science Education and Technology, 27(5):385-403, 2018.

19 Martin Monteiro and Arturo C Marti. Using smartphone pressure sensors to measure vertical velocities of elevators, stairways, and drones. Physics Education, 52(1):015010, 2017.

20 Martín Monteiro, Cecilia Cabeza, and Arturo C Martí. Exploring phase space using smartphone acceleration and rotation sensors simultaneously. European Journal of Physics, 35(4):045013, 2014.

21 Martín Monteiro, Cecilia Cabeza, and Arturo C. Marti. Acceleration measurements using smartphone sensors: Dealing with the equivalence principle. Revista Brasileira de Ensino de Física, 37:1303 -, 032015.

22 Rebecca Vieyra and Chrystian Vieyra. Physics toolbox suite, July 2019.

23 S Staacks, S Hütz, H Heinke, and C Stampfer. Advanced tools for smartphone-based experiments: phyphox. Physics Education, 53(4):045009, may 2018.

24 Tirra Hanin Mohd Zaki, Musab Sahrim, Juliza Jamaludin, Sharma Rao Balakrishnan, Lily Hanefarezan Asbulah, and Filzah Syairah Hussin. The study of drunken abnormal human gait recognition using accelerometer and gyroscope sensors in mobile application. In 2020 16th IEEE International Colloquium on Signal Processing 8 Its Applications (CSPA), pages 151-156. IEEE, 2020.

25 PM Harikrishnan and Varun P Gopi. Vehicle vibration signal processing for road surface monitoring. IEEE Sensors Journal, 17(16):5192-5197, 2017.

26 Braden J Limb, Dalon G Work, Joshua Hodson, and Barton L Smith. The inefficacy of chauvenet's criterion for elimination of data points. Journal of Fluids Engineering, 139(5), 2017.

27 Jonathan R Bradley and David L Farnsworth. What is benford's law? Teaching Statistics, 31(1):2-6, 2009. 